$\begin{array}{ll}\text { Italique } & \text { Italique } \\ \text { Poésie italienne de la Renaissance }\end{array}$

VIII | 2005

Varia

\title{
Scheda per il sonetto di Bembo a Paolo Giovio
}

\section{Andrea Donnini}

\section{(2) OpenEdition}

\section{Journals}

Edizione digitale

URL: http://journals.openedition.org/italique/120

DOI: 10.4000/italique.120

ISSN: 1663-4438

\section{Editore}

Librairie Droz

\section{Edizione cartacea}

Data di pubblicazione: 1 luglio 2005

Paginazione: 89-110

ISBN: 2-600-01024-6

ISSN: 1423-3983

Notizia bibliografica digitale

Andrea Donnini, « Scheda per il sonetto di Bembo a Paolo Giovio », Italique [Online], VIII | 2005, online dal 05 octobre 2009, consultato il 19 avril 2019. URL : http://journals.openedition.org/italique/120 ; DOI : 10.4000/italique. 120 
ANDREA DONNIN I

SCHEDA PER IL SONETTO

D I B EMBO A PAOLO GIOVIO 


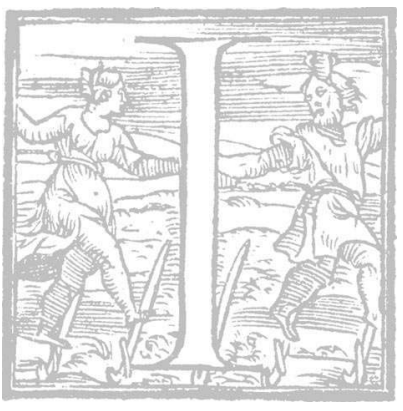

$L$ sonetto Giovio, che i tempi et l'opre raccogliete (Rime CXXXИIII) è tangibile suggello dell' amicizia fra Bembo e Paolo Giovio, nata nella Roma di Leone $X^{\mathrm{I}}$ e proseguita con assiduità variabile, ma senza interruzioni, nel corso degli anni. ${ }^{2}$ Bembo lo compone nella notte fra il 12 e il is dicembre I538 mentre si trova a Venezia. Ne informa Cola Bruno lo stesso I3 dicembre (Travi 1992, rr. 6-10 [dal ms. Bibl. Vat. "Chig. L VIII 304", c. 8Ir]]):

Questa notte ne ho fatto uno [sonetto] al Jovio per la causa che leggerete nella lettera di M. Carlo, che io vi mando, la qual mi rimanderete. Questo del Jovio si muterà. Ma vi priego me ne scriviate il parer vostro.

Cola Bruno risponde da Padova il I7:

[...]. Il sonetto di V. S. al Giovio è tutto bello et tutto puro, et a M. Flaminio et a me piace mirabilmente. Delli due versi, a me piace più questo: Che chiaro eternamente viverete. [...]. ${ }^{3}$

Bembo accetta il suggerimento e già il is dicembre annuncia all'amico il prossimo invio di una versione aggiornata (Travi 1994, rr. 2-7 [dal ms. Bibl. Vat. «Chig. L VIII 304», c. 87r]):

Di quel verso del sonetto, che più vi piaccia, già m'era io accordato con voi. Vederete il tutto ora un'altra volta, in alcuna parte cangiato da quello che egli era.

La lettera a Gualteruzzi allegata a quella a Cola del is dicembre deve coincidere (salvo modifiche, peraltro probabili, apportate dall'autore una volta che gli venne resa da Cola come richiesto) con quella che nell'epistolario bembiano risulta inviata da Venezia il 22 dicembre; in essa si accenna infatti al motivo della scrittura del sonetto al Giovio (Travi 1996 [dal ms. Bibl. Vat. "Chig. L VIII 304», c. 88]):

Feci a questi dì a Mons.r Jovio un sonetto per risposta di quello che mi scrivevate dintorno a gli amorevoli uffici che S.S. fa per me, e l'onorato testimonio che egli ne dà costì. Nol mandai, ché mi parve debole cosa, e basso. Per che mi venne in pensiero mutarne in parte il suggetto, e farlo di qualità che egli potesse mostrarsi con gli altri. Ora ne gli mando tuttadue, acciò vediate i capricci del mio pensiero. Se a voi parrà di dover dare il primo a Mons.r Jovio, dateglielo, molto nella sua 
buona grazia raccomandandomi. Ma l'altro per niente non mostrarete a persona alcuna, ma tenetelo rinchiuso nel vostro cassettino secreto. Questo dico acciò non paia che io torni al vomito dapoi gli due sonetti della penitenza che aveste. Nel vero tanto ho io un solo de' miei più freddi e leggier pensieri a donna del mondo, quanto avete voi alla reina di Scozia. Ma fingo, per aver da rimare. Tuttavia le male lingue arebbono materia da pettinarmi ora se vedessero quelli versi. Poscia si potranno por con gli altri, quando si ristamperanno costì. State sano.

\section{Alli XXII di Dicembre MDXXXVIII. Di Ven[ezi]a. Bemb[us] fr[ater]}

Il secondo sonetto non mando ora; manderollo un'altra volta.

Come ha avvertito Dionisotti " gli amorevoli uffici» che Giovio aveva intrapreso a Roma in favore di Bembo riguardavano la nomina a Cardinale. Bembo, tramite il Gualteruzzi, ringraziava Giovio delle cure che andava prendendosi per lui già l'II dicembre;' il I3 comunicava allo stesso Gualteruzzi il progetto di far eseguire da un valente maestro un proprio ritratto da donare all'amico, condizionato però dalla difficoltà nella scelta dell'artista (Travi 1993, rr. 25-29 [dal ms. Bibl. Vat. «Barb. Lat. 5693», c. I86r-v]):

Procaccerò di fare al tutto che Mons.r Jovio abbia un mio ritratto, ma ho molta fatica di Maestro che mi sodisfaccia. L'amore che S.S. mi dimostra, merita da me ogni grande opera e cura, non che questa. E se io il facessi di massiccio oro, non basterei a sodisfare al mio debito. Raccomandatemi a S.S. senza termine. [...].

Esistono quindi due lettere datate 13 dicembre relative alla gratitudine di Bembo nei confronti del Giovio. Suscita interesse il fatto che, nel riferire a Gualteruzzi i propri sforzi per procurare un ritratto di sé all'amico, Bembo non accenni neppure alla composizione del sonetto per lui testé avvenuta. Non è elemento di discrimine la differenza di destinatario perché tanto Cola quanto Gualteruzzi erano tenuti al corrente da Bembo sui progressi della sua attività letteraria. La questione sembra da dirimere guardando alla diversa provenienza delle due lettere. Il Barb. Lat. 5693 era parte del medesimo originario raccoglitore - il «Barberino I326», non un manoscritto pensato per la stampa ma un collettore di cui Bembo poteva in qualsiasi momento servirsi - ${ }^{6}$ poi smembrato; anche il Chig. L VIII 304 è un codice raccoglitore di lettere $e$ biglietti scritti in prevalenza a Cola Bruno e Carlo Gualteruzzi, arricchito di materiali diversi che ne hanno fatto «una di quelle cartelle dove viene raccolta la corrispondenza e tutto ciò che si intende non trascurare perché ricordo di particolari attività e scelte che non si sono potute continuare ma che si spera di 
riprendere un giorno». ${ }^{7}$ I due codici sono quindi di analoga natura; entrambi sono stati preparati in séguito a una più che probabile rielaborazione dei testi originariamente inviati; ma entrambi precedono il momento di organizzazione più sistematica in vista di un'edizione dell'epistolario e non sono stati reciprocamente armonizzati dall'autore. Per una migliore comprensione credo che occorra mettere da parte il dato puramente cronologico. Dall'insieme delle testimonianze sembra di capire che Bembo intendesse manifestare concretamente la propria gratitudine al Giovio e che la sua prima intenzione fosse quella di donargli un proprio ritratto. L'improvvisa ispirazione che gli consenti di comporre una rima in sua lode gli permise di rinviare il dono pittorico. ${ }^{8}$

Gli epistolari dello stesso Giovio e di Vittoria Colonna ${ }^{9}$ non recano informazioni ulteriori per la storia della rima in questione.

I. Il sonetto a Paolo Giovio è tramandato prevalentemente da testimoni d'autore. Si legge in tutte le raccolte delle Rime del Bembo note: $i$ manoscritti «I0245.I» della Oesterreichische Nationalbibliothek di Vienna, c. 52V (siglo W' il testo base, $\mathrm{W}$ seguito da lettere alfabetiche in progressione $i$ successivi stati; W ...z l'ultima fase elaborativa; mentre in contesti generici adotto $\mathrm{W})^{\mathrm{IO}}$ e «L. 1347-1957 (KRP. A. 19)» della National Art Library presso il Victoria and Albert Museum di Londra (L), c. s6r; ${ }^{\mathrm{II}}$ le edizioni a stampa Venezia, Giolito $1548(\mathrm{G}), c .48 \mathrm{r}^{\mathrm{I}}$ e Roma, Dorico I548 (D), p. $104 .{ }^{13} \mathrm{Il}$ testo base di $\mathrm{W}$ si fa risalire a prima dell'II novembre I54I; con esso infatti si identifica il manoscritto che con lettera datata a quel giorno Bembo invia in visione a Girolamo Quirini. ${ }^{14}$ Il testo base è stato progressivamente modificato da Bembo: in tre casi le variazioni sono datate; la data più alta è il 16 marzo I543, a margine di LVII I-2 («die Xvi. mens. | Mart. I543. | paulo post ort $\hat{u}$ | solis.»); quella più bassa è il 25 dicembre I545, a margine di Stanze 32, I-2 ( IIn mane nativitatis Dñi $1545 . »)$; il codice venne quindi rielaborato almeno sino al Natale del I545; ma probabilmente anche dopo. L e G condividono ciascuno uno stadio redazionale di $\mathrm{W}$ antecedente a quello piu avanzato: $\mathrm{L}$ si data fra il Is marzo I543 e il Natale IS45 dal momento che condivide la modifica a LVII I-2 ma non quella a Stanze $32, \mathrm{I}_{2}, \mathrm{I}^{\mathrm{I}}$ allo stesso modo si comporta $\mathrm{G}$, la preparazione del cui antigrafo è stata più precisamente datata da Paolo Trovato al febbraio $1544^{16}$

Autografo il testo del sonetto si legge nel codice «H 245 inf.» della Biblioteca Ambrosiana di Milano (M), c. 2r. ${ }^{17}$ Si tratta di un composito contenente una piccola raccolta di lettere di Bembo e corrispondenti, seguite da due di Pietro Aretino a terzi, non relazionabili direttamente con quelle che le precedono. $V i$ si trovano i documenti più importanti successivi al carteggio poetico fra Bembo e la Colonna, iniziato dalla marchesa che, per il tramite di Paolo Giovio, ${ }^{18}$ 
inviò a Bembo il sonetto a lui dedicato Ahi quanto fu al mio sol contrario il fato ${ }^{19}$ nella redazione che esordisce Se v'accendea il mio bel sole amato; Bembo rispose con il sonetto Cingi le costei tempie de l'amato (Rime CXXV) nella redazione con incipit Orna costei del sempre verde amato $^{20}$ inviato alla marchesa ancora attraverso il Comasco. ${ }^{21}$ La lettera della Marchesa da Ischia del 24 gingno, ${ }^{22}$ a c. Ir-v, è quella che ella scrisse a Giovio una volta ricevuto il responsivo bembiano; la lettera di Giovio del Is luglio, ${ }^{23}$ a c. br, è quella che egli scrisse al Bembo per accompagnare la lettera della Colonna. La lettera di Bembo del 16 settembre al Giovio, ${ }^{24}$ a cc. IIr-I2V, è la copia autografa con correzioni autografe della risposta alla stessa lettera della Marchesa. Ancora una copia autografa con correzioni autografe è la lettera, a c. sr, diretta da Bembo da Venezia a Vittoria Colonna il 2 luglio I533 per ringraziarla dellinvio di tre sonetti e di un suo ritratto. ${ }^{25}$ E opportuno parlare di copie con correzioni e non di minute, come fa invece Vecce descrivendo il codice, per il fatto che il testo base è una stesura in pulito e le correzioni sono recepite dal ms. Arch. Segr. Vat., "Fondo Borghese II 449» che è una raccolta organica di lettere a donne approntata da Bembo probabilmente prima della nomina cardinalizia ma dopo il 3 I agosto I535, data della lettera seriore (Travi I7IS); il codice era probabilmente parte di una più vasta silloge insieme al ms. Arch. Segr. Vat., "Fondo Borghese I 175" che contiene lettere a soli nomini la più tarda delle quali (Travi 2558) è datata 25 settembre $1546{ }^{26}$ Le circostanze certificano che le correzioni in $\mathrm{M}$ non appartengono al momento di stesura delle due lettere ma a un momento successivo di loro revisione "a freddo". Copia di calligrafo con varianti autografe è la lettera di Bembo a Sannazaro, Roma 24 dicembre $1517,{ }^{27}$ a c. 9r-v, che tiene dietro alla risposta di Sannazaro a Bembo a Roma del 9 gennaio $1518{ }^{28}$ alle cc. $7 \mathrm{r}-8 \mathrm{v}$, in copia autografa del Bembo medesimo. Anche la lettera di Bembo a Sannazaro è recepita dal ms. "Borghese I I75». Come ricorda Vecce, ${ }^{29}$ la corrispondenza con Sannazaro è in qualche modo legata a quella con la Colonna, perché, prima che a lei, al poeta napoletano Bembo chiese aiuto per il difficile governo della commenda gerosolimitana di Benevento assegnatagli nel ISI7. Si leggono in copia di calligrafo, ma senza correzioni dell'autore, la lettera di Bembo del 28 luglio I533 a Giovan Iacopo Leonardi ${ }^{\circ}$ (c. I3r) e la coppia di sonetti (CLXIV e CLXIII: alle cc. $3 \mathrm{r} e$ 4r) che è da associare per cronologia al sonetto al Giovio se, come sembra, con essi si debbono identificare $i$ «due sonetti della penitenza» cui accenna Bembo scrivendo a Gualteruzzi il 22 dicembre (Travi 1996). Senza correzioni marginali si legge anche il sonetto al Giovio; si tratta di una copia in pulito, autografa; una sola incertezza si ravisa al $v .7$ dove il sostantivo perle, ancora incompleto ("perl $)$, viene cassato e poi riscritto per intero di séguito nella stessa riga. Il foglio su cui il testo si trova scritto, in origine sciolto, è stato per analogia (ma impropriamente 
per la cronologia) allegato al registro gioviano-colonnese e applicato sul recto della c. 2 (che sul verso contiene l'indirizzo della lettera della Colonna a Paolo Giovio del 24 gingno I530) probabilmente da chi si trovò ad assemblare il fascicolo per conservare le carte in esso contenute.

Vecce, riferendosi al contenuto di $\mathrm{M}$, parla di «materiali che non solo denunciano una loro provenienza dall'angolo più riposto dello scrittoio bembino, ma che appaiono soprattutto legati assieme, ad opera dello stesso Bembo, in un unico fascio di memorie, di testimonianze della nascita d'una grande relazione intellettuale». ${ }^{3 \mathrm{I}}$ Mi sembra dubbio che sia stato proprio Bembo a comporre questo piccolo corpus. Non si tratta però di un aggregato disomogeneo. ${ }^{32}$ Come si è visto, la maggior parte dei documenti sono di pertinenza padovana. D'altronde conferma l'origine padovana del codice la sua provenienza dalla biblioteca di Gian Vincenzo Pinelli. ${ }^{33}$ La presenza di copie calligrafiche, talora con correzioni autografe, e di copie in pulito autografe con correzioni altrettanto autografe mi sembra che possa farpensare ai lacerti di una silloge di documenti apprestata forse nel momento in cui Bembo iniziava a progettare il riordinamento delle proprie lettere in vista di una loro edizione (la prima testimonianza esplicita di tale intenzione risale a prima del 28 novembre I535 come si comprende da una lettera datata a quel giorno inviata da Padova a Benedetto Varchi [Travi 1730$]) .{ }^{34} \mathrm{La}$ presenza di lettere altrui è poi in armonia con quanto accade ad esempio nello stesso Chig. L VIII 304 che contiene anche lettere di Lelio Torelli e di Andrea Alciato. E forse verosimile che Bembo in una prima fase andasse approntando un epistolario con significativi stralci degli scambi sostenuti con altre personalità. Mi risulta in ogni caso difficilmente spiegabile la presenza nel codice delle due lettere di Aretino: la prima ad Antonio de Leyva, autografa, Venezia 30 novembre I535, è una consolatio al dedicatario per la morte della figlia, ${ }^{35}$ la seconda ai Signori Veneziani, Venezia, 7 gingno I538, di materia politica, si trova in un foglio a stampa. ${ }^{36}$ La loro datazione non contrasta con quella degli altri documenti, per cui si potrebbe pensare che Bembo fosse entrato in possesso delle due lettere e che le conservasse fra le proprie; o che, in ragione della sincronia, venissero aggiunte al fascicolo dopo che questo fu assemblato. L'unico manoscritto apografo a tramandare il sonetto al Giovio è il settecentesco «MM rgI» della Biblioteca Civica «Angelo Mai» di Bergamo ${ }^{37}$ (p 65) che dipende, per le rime che contiene, e quindi anche per la nostra, da $\mathrm{D}$ o da sua derivata. Il testo rimase evidentemente consegnato alla stretta circolazione fra gli interessati.

Se nelle raccolte delle Rime il sonetto compare in una redazione pressoché concorde, la versione in $\mathrm{M}$ si distingue per le numerose varianti, cio che fa credere che essa sia anteriore a quella tramandata dagli altri testimoni: lo certifica il $v .4$ che legge in maniera diversa rispetto alla redazione preferita da Bruno, subito accolta da Bembo e a testo in tutta la restante tradizione. 
I tre testi poetici compresi in $\mathrm{M}$ sono stati pubblicati da Vecce e raffrontati con $\mathrm{D}$ in modo da rendere evidente lo scarto che li separa dall' ultima loro forma. ${ }^{38}$ Rinuncio all'edizione dei due sonetti penitenziali CLXIV e CLXIII estranei alle intenzioni di questo contributo; tramandati inoltre da cospicua tradizione che richiederebbe troppo spazio per essere qui illustrata e forse meriterebbe studio a parte. Offro l'edizione critica del nostro testo secondo la lezione di $\mathrm{M} ;{ }^{39}$ dispongo in apparato gli altri testimoni significativi secondo cronologia $([\mathrm{M}>]$ W' $>\mathrm{L} \mathrm{G}>\mathrm{W}$...z - D). La difficoltà di stabilire la cronologia relativa di

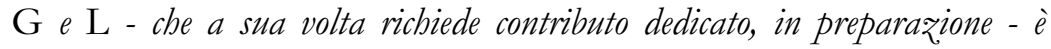
attenuata dal fatto che $i$ due testimoni leggono concordi, salvo un probabile errore, l'unico altrimenti riscontrato, di $\mathrm{G}$ al v. 6: ivi la lezione questo, pur non rendendo insensato il giro di frase, interrompe il forte iperbato istruito sui $v v$. 6-7 e relega il Donna gentil di $v .7$ a ruolo di apposizione esplicativa del v. 6; è pertanto facilior.

Giovio, che i tempi et l'opre raccogliete Quante ha degne di luce il secol nostro Con sì leggiadro et pellegrino inchiostro, Che chiaro et charo et sempre viverete,

Perché lo stile homai non rivolgete

A questa, novo in terra et dolce mostro,

Donna gentil, che non di perle et d'ostro,

Ma sol d'honor, anzi del cielo ha sete?

Questa risplenderà quasi bel sole

Tra gli altri lumi de le vostre carte

Et le rendrà via più lucenti et sole.

Quest'una ha in sé raccolto a parte a parte

Quanto in mill'anni a tutte dar non sòle

Di beltà, di valor natura et arte.

2] Del faticoso et duro secol nostro omnes. 3 Con...pellegrino] In così puro et sì lodato omnes. 4 et charo et sempre] eternamente omnes 6 questa] questo $G .7$ scrive perl e lo cancella; immediatamente di séguito: perle. 8 anzi del cielo] et di virtute $W$...z. (da T) D. 9 quasi] come omnes. Io Tra] Fra omnes. I I lucenti] gradite omnes. I 2 in sé raccolto] inseme quanto omnes. I3] Dar a mille ben nate a pena sòle omnes

2. La lezione del v. 4 fa ritenere che il testo di $\mathrm{M}$ sia quello letto da Cola Bruno la prima volta, il I3 dicembre; come si è visto (Travi 1994), Bembo accetta subito la preferenza sul luogo manifestata da Cola nella sua lettera del 17. L'ipotesi è coerente alla certa provenienza padovana delle carte del codice. Inoltre l'espediente paronomastico (chiaro et charo) ${ }^{40}$ non si registra altrove 
nella produzione bembiana in prosa e in versi e sembra poter essere ben proprio di una stesura primitiva.

Visto che sembra probabile che il foglio con il sonetto al Giovio sia quello letto da Cola Bruno successivamente all'invio del iz dicembre, occorre discutere il problema redazionale che fa sorgere la lettera a Gualteruzzi del 22 di quel mese, in cui Bembo informa l'amico di aver preparato due stesure del testo. La prima, giudicata «debole cosa», sarebbe stata infatti mutata in modo da renderlo «di qualità che egli potesse mostrarsi con gli altri», cioè nel canzoniere; questa più recente doveva essere di tema amoroso e coinvolgere una figura femminile nel ruolo di amata, per quanto astratta («fingo per aver da rimare»), se è definita «vomito» in confronto ai due sonetti di penitenza già partecipati a Gualteruzzi. Da quanto si legge, si dovrebbe pensare a un adattamento concentrato sui vv. 5-I4. Nella lettera Bembo annuncia la spedizione di entrambe le redazioni, dando ordine all' amico di mostrare eventualmente solo la prima al Giovio e custodire la seconda; $m a$ in extremis $c i$ ripensa e nel post scriptum annuncia a Gualteruzzi che non avrebbe inviato la versione "amorosa", ritenuta potenzialmente compromettente. Dionisotti ritiene che la redazione conservata sia quest'ultima visto che in essa "gran parte ha una donna». ${ }^{4 \mathrm{I}} \mathrm{Ma}$ anche nel sonetto in $\mathrm{M}$ ha larga parte una donna; e se la redazione in $\mathrm{M}$ può essere quella letta da Cola Bruno, visti gli sviluppi successivi, che modificano ampie parti ma non l'architettura del pezzo, occorre pensare che del sonetto non sia sopravvissuta proprio la redazione modificata. Nel momento in cui Bembo decideva di non mandarla a Gualteruzzi in fin dei conti ne impediva la circolazione; potrebbe anche darsi che in séguito vi avesse rinunciato.

Riguardo allidentificazione della donna di cui Bembo chiede a Giovio di scrivere, è decisivo sapere che per la sua elezione al cardinalato anche la Colonna si era adoperata presso il Papa, sin dal $15366^{42}$ Lo testimoniano anche due lettere di Bembo a lei: nella prima, datata Venezia Is marzo I539 (Travi 2024), egli la ringrazia per le fatiche intraprese in suo favore e, accennando all'opposizione di alcuni detrattori, la rassicura sul fatto che nessuna delusione gliene verrà in caso la nomina cardinalizia (già in pectore dal 20 dicembre 1538$)^{43}$ non si realizzi; ${ }^{44}$ la seconda, datata Venezia 4 aprile I539 (Travi 2039), attribuisce alla Marchesa gran parte del merito dell'avvenuta nomina. ${ }^{45}$ Stanti le circostanze, è ragionevole, come ha fatto Dionisotti, identificare con la Colonna la donna della quale nel sonetto Bembo suggerisce a Giovio di scrivere, ${ }^{46}$ anche per gli stretti rapporti che l'avevano legata al Giovio $^{47}$ e, nel caso particolare, a Bembo attraverso Giovio. Analoga proposta aveva fatto anche l'Ameyden. ${ }^{48}$

Accettata questa identificazione, risulta ancora più difficile credere che quella sopravvissuta sia la redazione "amorosa" non spedita a Gualteruzzi, in 
merito alla quale Bembo riconosceva di aver adottato moduli corrivi di lode. Si ritiene infatti che dovendo parlare della Colonna altro sforzo compositivo avrebbe intrapreso. Assume poi diverso significato il v. 9 del sonetto, che si può intendere come il riverbero sulla poetessa della mitologia solare da lei associata alla figura del marito defunto.

Elisabetta Quirini ne sarebbe stata il soggetto secondo Seghezzi. ${ }^{49}$ La proposta è coerente con la collocazione del sonetto, che, in tutte le raccolte che lo testimoniano, segue la serie per la Veneziana (Rime CXXXII-CXXXVII). La dedica della serie si trova apertamente dichiarata nell'indice di $\mathrm{D}$ per intervento del curatore Carlo Gualteruzzi, ${ }^{5 \circ}$ ma non lo è in G. Seghezzi, per l'identificazione, aveva come garanzia le informazioni in D. Di fatto occorre ammettere che il testo di per sé non consente di capire che si sta parlando della Colonna; tanto che Sansovino nelle parche note all'edizione delle Rime di Bembo da lui curata $^{\text {I }}$ (basata su $\mathrm{G}$ ) poteva introdurlo in questo modo (c. $4 \mathrm{~V})$ : «Scrive a Monsignor Giovio Historico celebratissimo, et l'invita a scriver della sua donna nelle sue Historie». Anche contemplando fra le ipotesi la distrazione di Sansovino, pare logico considerare che se tanto palese fosse stata l'identificazione al suo tempo, proprio per distrazione l'avrebbe riferita. Forse, collocando il sonetto, Bembo ba tenuto a osservare la cronologia relativa delle rime epistolari. Ma si potrebbe anche pensare che nel rivedere il pezzo per l'inserimento nel canzoniere, rinunciando alla versione di impianto modificato, egli ne preferisse una collocazione per cosi dire "discreta" (una aperta dichiarazione sarebbe stata la collocazione del sonetto ad esempio dopo i tre, CXXVсхХVII, alla Marchesa di Pescara esplicitamente indirizzati), intesa a rispettare la peculiarità del ruolo di Giovio nel rapporto con la Marchesa: che di propria iniziativa aveva iniziato il carteggio culturale con lui, ma occultandosi dietro il Comasco, a preservare cosi la natura di un'amicizia «sorretta sempre [...] dalla predisposizione, che entrambi [Bembo e la Colonna] avevano, a una virile prudenza temperata dal capriccio». ${ }^{52}$

Il suggerimento che nel sonetto Bembo dà a Giovio pone un altro problema di coordinazione. Bembo infatti era già a conoscenza delle molte buone parole spese nelle sue opere da Giovio per la Colonna e gliene rendeva atto nella lettera I6 settembre I530 (Travi II45, rr. 37-39: "Ella sicuramente è quella gran Donna che voi avete e al mondo più d'una volta dipinta con l'onorato inchiostro delle vostre prose, e a me molte fiate dissegnata con le parole.»). ${ }^{33} \mathrm{Si}$ potrà intendere come un auspicio post eventum, ma non sarà ozioso cercare di capire se invece Bembo non si riferisca a qualche iniziativa gioviana in corso. La prima quartina del sonetto allude all'opera di storiografo del Giovio; si potrebbe quindi genericamente pensare che Bembo lo esortasse a menzionare ampiamente la Colonna nelle Historiae sui temporis. ${ }^{54}$ Potrebbe essere un'ipotesi meno generica la biografia che Giovio andava scrivendo del marito 
della Colonna, a cui la poetessa si riferiva nel sonetto Di quella chiara tua serbata fronde ${ }^{55}$ dedicato proprio a Giovio; ma la biografia, pronta per la stampa solo nel 1548 , era già in preparazione da molto tempo ${ }^{56}$ visto che la prima circolazione del citato sonetto della Marchesa è da assegnare ai dintorni del I530. ${ }^{57}$ E forse piu probabile che Bembo intenda alludere ai tre Dialogi de viris et foeminis aetate nostra florentibus, di cui vide la copia che, nel 1529, Giovio ne portò con sé a Bologna, ${ }^{58}$ cercando di farli stampare con l'aiuto di Isabella d'Este, e di cui, fallito quel primo tentativo, di nuovo progettava l'edizione nel I535. ${ }^{59}$ L'invito potrebbe quindi leggersi come un indiretto auspicio per la conclusione e la pubblicazione dell'opera, che in Vittoria aveva il suo centro e in suo onore si chindeva con un ampio elogio, «forse il piu ampio elogio scrittole da un contemporaneo». ${ }^{60}$

3. Il sonetto non ha riscosso fortuna di recente, visto che non si legge nelle più aggiornate antologie di lirica cinquecentesca. ${ }^{61}$ Sotto il profilo letterario non è dei migliori di Bembo. É esponente medio dei sonetti di carattere epistolare, forse collocabile - nella tassonomia proposta da Domenico Chiodo - fra gli «attestati di appartenenza alla comunità dotta». ${ }^{62}$ La medietà della prova è rivelata dall'impiego di moduli e temi già da Bembo sperimentati. L'indirizzo al dedicatario in apertura è tipico della poesia di carattere relazionale; l'ampliamento tramite relativa che occupa la prima quartina è sperimentato da Bembo in alcuni sonetti epistolari (LXXIV, CXXII, CXXXII) $e$ in alcuni di morte (CXLIV, CXLV, CXLVI, CXLVII, CLV) nonché in altri allocutivi (LXXXIV, LXXXVIII, CI, CII, CXVI, CXVIII). Qui l'autore argomenta l'allocuzione sintetizzando gli elementi di merito del destinatario e implicitamente giustificando la sua elevazione poetica. Allude (vv. I-2) in maniera generale all'attività di storico di Giovio; nel riuso di Petrarca, Tr. fam. $55-56$ «Tuchidide vid'io, che ben distingue / i tempi e' luoghi e l'opere leggiadres ${ }^{63}$ concentra però l'attenzione sul classico binomio opera et dies. Indica (vv. 3-4) la ragione della fama imperitura dell'opera gioviana nelle sue qualità di stile: definito prima sì leggiadro et pellegrino e poi così puro et sì lodato. In termini simili alla prima versione Bembo definisce lo stile del Giovio in una lettera a Papa Clemente VII da Padova 7 aprile I530 (Travi I076 r. 25): «Il che [le feste occorse in occasione dell'incoronazione di Carlo $V$ imperatore] avere udito, in vece d'averle vedute, mi gioverà rimettendomi poi alla imagine e sembianza di loro, che col suo dotto e leggiadro stile ci ritrarrà nelle sue istorie Mons. Iovio.». La prima coppia di aggettivi è utilizzata da Bembo in accezione galante nel madrigale Né le dolci aure estive (Asolani II VI), 4 «Donna passar leggiadra et pellegrina»: ${ }^{64}$ desunta da Petrarca Tr. Et. 85 «E tra l'altre leggiadre e pellegrine» (e cfr. Rvf. 2I3, 5 «leggiadria singulare et pellegrina»). ${ }^{65}$ Una coppia simile Bembo usa infine in riferimento allo stile di Petrarca poeta 
d'amore in Rime XXXVI I2 «[...] leggiadro et onorato stile». Il cambiamento in cosi puro et si lodato assimila il dettato ai più antichi sonetti CXXXIX 7 «tutto'l suo puro e non caduco inchiostro» (IS36) e LXXV \& "ral buon Lombardo, il cui lodato inchiostro» (ISIO-ISII).

Il discorso prende, nella seconda quartina, una piega diversa, e prosegue con un suggerimento concreto: quello di scrivere di una donna virtuosa, che si è detto poter essere Vittoria Colonna. Bembo si era indirettamente proposto quale suggeritore per l'opera di storico e di biografo di Giovio in una lettera a Giambattista Ramusio dell'8 dicembre I522. ${ }^{66} \mathrm{Ma}$, soprattutto, qui sembra ricordare il sonetto al Gualteruzzi (Rime CXXVIII I-8: "Carlo, dunque venite a le mie rime / Vago di celebrar la donna vostra, / ch'al mondo cieco quasi un sol si mostra / di beltà, di valor chiaro e sublime? // E non le vostre prose elette e prime, / come gemma s'indora o seta inostra, / distendete a fregiarla, onde la nostra / e ciascun'altra età più l'ami e stime?»), composto fra il I530 e il I535. ${ }^{67}$ Ivi il dedicatario, seppur con una praeteritio, è invitato a scrivere in prosa della propria donna, che è connotata da tratti di eccellenza simili a quelli delineati per la Colonna ( 3 quasi un sol si mostra - 9 risplenderà quasi bel sole; 4 di beltà, di valor - I4 Di beltà, di valor). Tratti analoghi tornano nel sonetto a Pietro Aretino (Rime, Rifutate IV), composto tra il novembre 1536 e il febbraio $1537^{68}$ sulle tracce di quello a Gualteruzzi: si tratta anche in questo caso di una praeteritio con cui Bembo declina l'invito a scrivere in lode di Angela Serena adducendo la qualità dell'opera del richiedente che è riuscito a conferire alla donna amata stato di eccellenza non valicabile (I2-I3 La quale [Angela Serena] oggi risplende tra le prime / per voi - 9 Questa risplenderà quasi bel sole; $I 3$ sì come novo $\mathrm{e}$ dolce mostro - 6 novo in terra et dolce mostro; I4 di beltà, di valor chiaro e sublime - Di beltà, di valor natura et arte). ${ }^{69}$

Il vero e proprio consiglio si trova ai vv. 5-6. Qui (la transiżione - v. 5 - mima Rvf. $7 I, 8$ «a voi rivolgo il mio debile stile») si opera il passaggio del centro focale dal dedicatario alla donna. Qualcosa di analogo accade anche nel sonetto di Giovanni Guidiccioni Giovio, com'è che fra l'amaro pianto: che precede cronologicamente quello di Bembo e, pur diretto a Giovio, è in realtà in tutto una lode alla Colonna e alle sue rime funebri. ${ }^{70}$ I motivi (vv. 6-I4) che rendono degna la donna del privilegio di essere oggetto di attenzione da parte di Giovio fanno brillare solo di luce riflessa il dedicatario: evidentemente in possesso di capacità congrue rispetto a un soggetto tanto elevato. Auspicio legato all' accoglimento dell'invito è il supplemento di fama che Giovio otterrà accogliendolo: la donna di cui tratterà, novo in terra et dolce mostro $(v$. 6, da Rvf. 347, 6 «o de le donne altero e raro mostro»), spiccherà infatti come un sole fra le altre personalità ricordate (vv. 9-IO) e le carte gioviane saranno quindi via più lucenti (> gradite) et sole (come le «rime» funerarie della 
Colonna, "sì soavi e si conteste, / ch'a l'età dopo noi solinghe andranno» [Rime сххVI 5-6]). L'eccellenza della Colonna è delineata secondo abituali connotati: la sua aspirazione a valori morali e celesti piuttosto che agli agi delle ricchezze materiali (vv. 7-8), per cui Bembo, che aveva già sviluppato it concetto nei riguardi di Trifon Gabriele (Rime CXXII I-4 «Trifon, che 'n vece di ministri e servi, / di loggie e marmi e d'oro intesto e d'ostro, / amate intorno elci frondose e chiostro / di lieti colli, erbe e ruscei vedervi»), si avvale ancora del già citato Rvf. 347, 4 «et d'altro ornata che di perle o d'ostro» e di Rvf. 335, 6 «sì come a cui del ciel non d'altro calse» la cui memoria è forse combinata con Rvf. 263, 5-6 «vera donna, et a cui di nulla cale, / se non d'onor [...]»; il cumulo di bellezza e virtù che la natura ba creato in lei (vv. I2-I4), percuifida nuovamente su Petrarca, Rvf. 193, I2-I4: «Allorinseme, in men d'un palmo, appare / visibilmente quanto in questa vita / arte, ingegno et Natura e'l Ciel pò fare», e insieme ancora ricorda se stesso per il v. I4: Rime XX I4 «Bellezza, leggiadria, natura et arte». Anche l'auspicio di fama per le carte gioviane (vV. 9-II) tradisce il riuso di un classico tema, la donna sole fra le stelle, già impiegato da Bembo, oltre che nei citati sonetti a Gualteruzzi (v. 3) e Aretino (v. I2), anche in Rime XVIII 4-5 "[...] la chiara e gran virtute vostra, / ch'è quasi un sol, ch'ogni altro lume adombra»; e variamente fruito, seppur con fini diversi, da Petrarca (cfr. ad es. Rvf. II9, 69-72; in chiave amorosa: Rvf. 72, 40-4I; Rvf. I8I, 9; Rvf. 218, I-4; Tr. Cup. III 133).

L'apporto petrarchesco è ambivalente: sia concettuale che verbale. Le citazioni di Rvf. 347 sono poi confermate da ricordi di carattere metrico: se lo schema del sonetto petrarchesco differisce nelle terzine ( $C D E C D E$; questo $C D C D C D)$ Bembo riprende però le parole rima in -ostro (Rvf. 347, I nostro, 4 ostro, 5 mostro, 8 [i] nchiostro) nonché lo stratagemma della rima identica (sole; in Rvf. 347 volsi, vv. I0, I3).

Il campo metaforico che attraversa il sonetto è quello della luce: sottolinea la chiara fama dell'opera dello storico, e della donna; nonché il lustro che deriva dall'adeguata celebrazione di eventi e personaggi. La rielaborazione attenua il motivo modificando completamente il v. 2 (si indica l'opera dello storico non più nel raccogliere solo notizie degne di essere tramandate - -[...] $i$ tempi et l'opre raccogliete / Quante ba degne di luce il secol nostro» - ma tutte quelle del tempo presente, definito faticoso e duro: dittologia che mi risulta utilizzata prima solo in Ariosto, Orlando Furioso XLIII I86, 4 e Sannazaro, Rime disperse I 63, mentre riscontro in Rvf. I84, 8 un faticosa et vile; ma secol duro usava Bembo già in Rime CXXI I4) e il lucenti al v. II con gradite. Ma si mantiene la caratteristica solarità della donna: che, come si è visto, non sembra casuale.

L'impressione che si ricava dall'analisi è che Bembo abbia scritto i vv. 6-8 e I2-I4 utilizzando piuttosto meccanicamente la struttura compositiva fornita da 
Rvf. 347; che ad essi abbia giustapposti $i$ vv. I-s e con $i$ vv. 9-II abbia realizzato il necessario nesso per dare tenuta al componimento. Anche i temi utilizzati per la lode della donna gentile sono piuttosto comuni; per essi sembra calzante nella sostanza il gindizio di corrività formulato dallo stesso Bembo nel rivolgersi a Gualteruzzi. Tale valutazione si riferiva alla versione "amorosa" del sonetto. Non credo che Bembo per parlare della Colonna si sarebbe messo in questo ordine di idee. Se - a stento - è possibile ravvisare qui qualche ricordo dei sonetti alla Colonna apertamente dedicati (per il tema della concordia in lei di bellezza e virtù cfr. ad es. Rime CXXVI 4 " "[...] pensier' santi, et ragionar celeste»; e CXXV $5-8$ «e se 'n donna valor, bel petto armato / d'onestà, real sangue onorar vuoi, / onora lei, cui par, Febo, non puoi / veder qua giù, tanto dal ciel l'è datos; per la capacità di dare lustro al secolo in cui vive, cfr. Rime CXXVII I "Caro et souran de l'età nostra honore, / Donna d'ogni virtute intero exempio»), nel complesso pare che questi presentino un superiore grado di rielaborazione e di riappropriazione della tradizione culturale cui si riferiscono e nell'insieme un diverso e migliore sforzo di elaborazione.

Nella storia del sonetto alcuni elementi appaiono incongrui. Bembo racconta di averlo composto nella notte per improvvisa ispirazione, come già aveva fatto per il sonetto a Vettor Soranzo (Rime CLIV): ${ }^{71}$ ciò che può far pensare a un topos piuttosto che a una reale occorrenza. Bembo invita Giovio a scrivere della Colonna, ma questi se ne era già ampiamente occupato. E soprattutto sceglie temi piuttosto comuni se non banali, e una loro usuale declinazione per delineare l'eccellenza nientemeno che della Marchesa di Pescara. Tali occorrenze, insieme alla conduzione artistica del pezzo, suggeriscono un'illazione. Bembo, scrivendo a Gualteruzzi il 22 dicembre I538, potrebbe aver fornito una ricostruzione alterata del suo percorso compositivo, che potrebbe essere stato in realtà il seguente: l'autore, desideroso di donare qualcosa a Giovio e non riuscendo a farsi fare un ritratto, potrebbe aver riciclato un sonetto amoroso, forse già scritto o in corso d'opera, e probabilmente in rapporto con $i$ sonetti a Gualteruzzi e Aretino, riutilizzando i motivi encomiastici come giustificazione per linvito al dedicatario a scrivere della donna. Nel nuovo contesto questa si deve intendere essere Vittoria Colonna. Se le cose fossero andate cosi, la relativa sufficienza nella redazione della rima da parte di Bembo si capirebbe meglio: nei rispetti del Giovio come un portato della fretta con cui fu redatto il componimento; e nei rispetti della Colonna per il fatto che la sua presenza sarebbe un indotto e non la realizzazione di un progetto. Certo la mancanza di documenti impedisce di avallare un'ipotesi che prevede l'orchestrazione consapevole da parte di Bembo di una menzogna nei confronti dell'amico Gualteruzzi. Occorre peraltro menzionare la riserva per cui la lettera 22 dicembre potrebbe non coincidere con quella effettivamente letta da Gualteruzzi, visto che il suo testo nel «Chig. L VIII z04» è probabile esito di 
Bembo e Giovio

una revisione. Il fatto che Bembo comunque vi nomini la redazione "amorosa" significa che in ogni caso egli era intenzionato a far conoscere all'amico questo retroscena: se infatti il rischio che Gualteruzzi si lasciasse sfuggire qualcosa gli fece decidere in extremis di non inviare il testo della versione "amorosa", tale rischio permaneva anche nei riguardi della notizia di averla composta; per evitarlo Bembo avrebbe dovuto tacere del tutto la sua esistenza. Il fatto che non abbia scelto questa soluzione è prova che riferire il particolare era per lui essenziale. La volontà di mantenere la notizia potrebbe averlo indotto quindi a modificare nel racconto l'ordine delle cose: salvaguardando una forma di cautela nei confronti del Giovio, e ottenendo insieme un'implicita giustificazione, di fronte a Gualteruzzi ed eventualmente a chiunque altro, della permanenza nel testo di una seppur tenue ispirazione amorosa, retrocessa da momento autonomo e relativamente autentico a frutto di una contingente revisione necessaria per l'inserimento del pezzo di partenza fra le Rime.

Andrea Donnini 


\section{ANDrea Donnini}

Desidero ringraziare il dott. Domenico Chiodo e il dott. Emilio Torchio che hanno letto queste pagine. Con particolare gratitudine mi rivolgo al prof. Roberto Tissoni che ha esaminato il lavoro discutendolo con me analiticamente.

Si utilizzeranno le seguenti abbreviazioni:

Dionisotti $=$ PIetro Bembo, Prose della volgar lingua - Gli Asolani - Rime, a cura di C. Dionisotti, Milano, TEA, 1989 (ristampa dell'edizione Torino, UTET, 1966). Le rime del Bembo sono citate secondo questa edizione.

Travi $=$ Pietro Bembo, Lettere. Edizione critica a cura di E. Travi, Bologna, Commissione per i Testi di Lingua, 1987-1993, 4 volumi.

I. Dionisotti, p. 6rg. Giovio fu a Roma almeno dal I 5 I4, anno in cui il suo nome compare come insegnante di filosofia morale allo Studio romano (T. C. Price Zimmermann, Giovio, Paolo, in Dizionario Biografico degli Italiani, Roma, Istituto dell'Enciclopedia italiana, vol. 56, [200I], p. 430b). Resta testimonianza della sua frequentazione con Bembo anche in Ludovico ArIosto, Satire, VII I 27-29.

2. Poche sono le lettere scambiate direttamente fra i due: tre di Bembo (7 aprile I 530 [Travi I077], 29 maggio I 530 [Travi I094], I6 settembre I 530 [Travi I I45]); due di Giovio (I5 luglio i 530 [PAOLO GIOvIO, Lettere, a cura di G. G. Ferrero, ad iniziativa dello Istituto Storico Italiano per l'età moderna e contemporanea, vol. I, I 14 4-I 544 , Roma, Istituto Poligrafico dello Stato, Libreria dello Stato, i 956 , vol. II I $544-$ I 552 , ibid., i 958 : n. 35 , vol. I p. I 25$]$ e [marzo] 1539 [ibid., n. 94, vol. I p. 2 I 5 ]). La prima menzione di Giovio in lettere di Bembo risale al 6 ottobre i 22 (Travi 430); l'ultima al i 3 gennaio is 39 (Travi 2008). La prima menzione di Bembo nelle lettere di Giovio risale al i 534 - I 535 (PAolo Giovio, Lettere, n. 6o, vol. I pp. 173-77); l'ultima di Bembo vivo all' I I gennaio i 547 (PAolo Giovio, Lettere, n. 245 , vol. II p. 65). Solo una parte del rapporto fra i due è quindi supportato dalle testimonianze epistolari. Nella maggior parte dei casi Bembo saluta o comunica qualcosa all'amico tramite altre persone; più estrinseche le menzioni gioviane.

3. Delle Lettere da diversi Re, et Principi, et Cardinali, et altri buomini dotti a Mons. Pietro Bembo scritte, primo volume di nuovo stampato, riveduto et corretto per Francesco Sansovino, In Venetia, Appresso Fran. Sansovino et compagni, i 560 (rist. anast. a cura di Daria Perocco, ISR-Ferrara / Sala Bolognese, Forni, I985), V 65.

4. Dionisotti, p. 6r9; C. Dionisotti, Appunti sul Bembo e Vittoria Colonna, in Miscellanea Augusto Campana, Padova, Antenore, I98 I, vol. I, pp. $258-86$ (p. 272); ora in Id., Scritti sul Bembo, a cura di C. Vela, Torino, Einaudi, 2002, pp. I I 5-40 (p. I 28).

5. Travi 1991 I, rr. 4-9 (da ms. Bibl. Vat. «Barb. Lat. $5693 »$, c. I 84 r): «Direte a Mons. ${ }^{\mathrm{r}}$ Jovio che ogni dì veggo più chiaro il buono e vivo amore che S.S. mi porta, e che io gliene rendo quelle maggiori grazie che io posso. Serberò nel mezzo del mio cuore l'infinito e caro obligo che io gli sento del bello e onorato testimonio che S.S. fa di me in quel teatro del mondo; raccomandatemegli senza nessun termine. [...]».

6. E. Travi, Pietro Bembo e il suo Epistolario, «Lettere Italiane», a. XXIV (1972), n. 3, p. 295; Travi I p. XXX.

7. Travi, Pietro Bembo e il suo Epistolario cit., p. 296; Travi I p. XXX. Sugli allegati non epistolari si vedano: C. Vecce, Bembo, Boccaccio, e due varianti al testo delle «Prose», «Aevum», a. LXIX (1995), n. 3, pp. 52 I-3 I ; Id., Bembo e Poliziano, in Agnolo Poliziano poeta scrittore filologo, Atti del Convegno Internazionale di Studi, Montepulciano 3-6 novembre 1994, a cura di V. Fera e M. Martelli, Firenze, Le Lettere, I998, pp. 477-503. 
8. Risale al 1539 il ritratto di Bembo Cardinale dipinto da Tiziano oggi conservato alla National Gallery of Art di Washington, sul quale si può consultare la scheda di D. A. Brown, in Tiziano, Palazzo Ducale, Venezia - National Gallery of Art, Washington, Venezia, Marsilio, I990, p. 238. Nella lettera a Girolamo Quirini, Roma 30 maggio I 540 (Travi 2 I9I rr. I6-20), Bembo scrive all'amico: «[...]. Renderete parimente grazie a M. Tiziano del dono del mio secondo Ritratto; il qual Ritratto io volea scrivervi, come io veduto l'avessi, che gli fosse pagato come era conveniente. Ora che la sua cortesia vuole che io gliene resti ubligato, così sarà, e farò un dì alcuna cosa anco io per lui.». Evidentemente Tiziano eseguì due ritratti di Bembo a poca distanza l'uno dall'altro. Entrambi dovevano essere su commissione, ma solo di uno l'artista chiese il pagamento. È molto probabile che entrambi raffigurassero Bembo in veste cardinalizia. Uno dei due è quello conservato a Washington. Giovio nel suo Museo aveva un ritratto di Bembo Cardinale di cui si conserva agli Uffizi la copia eseguita da Cristoforo dell'Altissimo (riproduzione in Gli Uffizi. Catalogo Generale, a cura di L. Berti, Firenze, Centro Di, I979, p. 6 I I , fig. Ic 60). Nulla si può dire con certezza in proposito ma sorge spontaneo supporre che uno dei due ritratti del Bembo Cardinale eseguiti da Tiziano potesse essere stato donato al Giovio; una matrice tizianesca nel ritratto di Bembo del museo Giovio è riconosciuta da G. Coggiola, Per l'iconografia di Pietro Bembo, «Atti del Reale Istituto Veneto di Scienze e Lettere ed Arti», LXXIV (I9I4-I 9I 5), p. 5 I I n.: «[...] il ritratto gioviano del nostro porporato doveva, forse, discendere dal primo ritratto tizianesco successivo alla elevazione di Bembo al cardinalato». Notizie sulliconografia bembiana anche in C. Damianski, Liceità e pratica dell' imitazione nelle Prose. Bembo e il recupero dell'antico nel primo Cinquecento (letteratura e arte), in Prose della Volgar Lingua di Pietro Bembo, Gargnano del Garda (4-7 ottobre 2000), a cura di S. Morgana, M. Piotti, M. Prada, Milano, Cisalpino, 2000, pp. 617-54 (pp. 635-54).

9. Vittoria Colonna, Carteggio, raccolto e pubblicato da E. Ferrero e G. Müller. Seconda edizione con Supplemento raccolto ed annotato da D. Tordi, Torino, Loescher, I 892.

ı. Sul codice si veda ora Pietro Bembo, Stan₹e, edizione critica a cura di A. Gnocchi, Firenze, Società Editrice Fiorentina, 2003, pp. LIV-LVII, con la bibliografia pregressa.

I I. Su cui A. Gnocchi, Un manoscritto delle Rime di Pietro Bembo (Ms. L. 1347-I957 KRP. A. I9 del Victoria and Albert Museum di Londra), «Studi di Filologia Italiana», LX (2002,) pp. 217-36. Molte informazioni sono riutilizzate in PIEtro Bembo, Stanze, pp. LIX-LX.

I 2. Pietro Bembo, Stanze, pp. LVII-LIX, con la bibliografia pregressa.

i 3. Pietro Bembo, Stan₹e, pp. LXI-LXVI, con la bibliografia pregressa.

I4. Travi 2297 rr. 20-28: «Sopra le quali Rime voglio che sappiate che, avendomi già detto M. Carlo più d'una volta che egli crederia che fosse bene che si facesse una impressione di tutte insieme, ciò è di quelle de gli Asolani e delle altre, io laudai questa openion sua, e ordinai che ne fosse trascritta una copia di tutte insieme, traponendo quelle de i detti Asolani con le altre, secondo che mi parea star bene. E così fu fatto. La qual copia si truova ora in mano di esso M. Carlo, il qual partendo di Roma per seguitar la Corte di Nostro Signor, e appresso per venirsene costà, mi chiese di portarla seco. [...]».

I5. Gnocchi, Un manoscritto cit., p. 225.

I6. P. Trovato, Per la storia delle Rime del Bembo, «Rivista di Letteratura italiana», IX, 3 (I99I), pp. 465-508 (pp. 474-78).

17. Il codice è cartaceo, composito, tutto del sec. XVI, mm. 350x240, cc. II, I-17, II' così numerate modernamente a matita. Appare a volte una precedente numerazione a penna (dei singoli documenti) di cui rimangono visibili i numeri I (c. I), 2 (c. 2), 3 (c. 7), 4 (c. 9), 


\section{ANDrea Donnini}

6 (c. I I), 8 (c. I 3). Bianche le cc. Ir, II, 3v, 4V, 7v, 8r, I or, I 3V, I4, I6v. I documenti, di formati diversi, sono stati incollati su supporti cartacei della misura data. Anepigrafo. Legatura moderna in pergamena molle. Il suo contenuto si trova descritto nell'Inventario Ceruti dei manoscritti della Biblioteca Ambrosiana, Trezzano sul Naviglio, Etimar, I979, 5 voll., II, p. 402; nell'Iter Italicum di Kristeller (I p. 294); in C. Vecce, Paolo Giovio e Vittoria Colonna, «Periodico della Società Storica Comense», 54 (1990), pp. 65-93 (p. 91, n. 85) che cita C. Ranieri, Censimento dei codici e delle stampe dell'epistolario di Vittoria Colonna, «Atti e Memorie dell'Arcadia», s. III (1980-I98I), p. 273; è stato fruito sia nell'edizione dell'epistolario del Giovio (Giovio, Lettere, I, p. 34), sia in quella del Carteggio della Colonna (passim); oltre che in quella dell'epistolario bembiano (Travi I p. XVI) e dell'epistolario di Sannazaro (IACOPO SANNAZARO, Opere volgari, a cura di A. Mauro, Bari, Laterza, I96I, pp. 409-Io); si trova menzionato da P. De Montera, La Beatrice d'Ambroise Leone de Nola; ce qui reste d"un «Beatricium» consacré a sa gloire, in Mélanges de Pbilologie, d'bistoire et de littérature offerts à Henri Hawvette, Paris, Les Presses Françaises, 1934, p. 191, n. 2.

I8. Lettera Padova 7 aprile I 530 (Travi 1077): «Ieri solamente ebbi, R.do Mons. mio, le lettere di V.S. delli XVII del passato, mandatemi insieme col bello e leggiadro Sonetto della Marchesa di Pescara, e con la lettera che ella, mandandovi il Sonetto, a voi scrive. [...]».

19. Vittoria Colonna, Rime, a cura di A. Bullock, Bari, Laterza, 1982, A7i.

20. Consegnata ai manoscritti: Bibl. Ambr., «I 56 inf.» c. I6r, Bibl. Med. Laurenziana, «Ashburnham i I 53» c. 3 Ir; Bibl. Naz. Marciana, «It. IX 300» («6649») c. 4r, Bibl. Naz. Vittorio Emanuele III, «XIII G 43» c. 44v, Bibl. Vat., «Vat. Lat. 5 I 70 » c. 8 5r, Bibl. Vat., «Vat. Lat. $\{172 》$ c. $2 \mathrm{r}-\mathrm{v}$.

21. Lettera Padova (di Villa Bozza) 29 maggio I 530 (Travi I094): «Mando a V.S. la risposta, fatta da me quando Dio ha voluto, al bellissimo Sonetto della S.ra Marchesa di Pescara. $[\ldots] »$.

22. Vittoria Colonna, Carteggio, XLI.

23. Paolo Giovio, Lettere, n. 35, vol. I pp. i $25-26$.

24. Travi II 45 .

25. Vittoria Colonna, Carteggio, LVIII; Travi I 50 I.

26. Travi I p. XXXIV.

27. Travi 387 .

28. Iacopo Sannazaro, Opere volgari, pp. 3i 8-i 9 .

29. Vecce, Paolo Giovio cit., p. 84.

30. Travi I 506.

31. Vecce, Paolo Giovio cit., pp. 90-91.

32. Travi, Pietro Bembo e il suo Epistolario cit., p. 291, n. I 4 elenca il codice nel gruppo di quelli con lettere sparse.

33. Vecce, Paolo Giovio cit., p. 9I, n. 85. Vecce ricorda altri importanti manoscritti bembiani di provenienza Pinelli conservati all'Ambrosiana: «i codici H 246 inf. (= S.P. II ıoo; versi 
spagnoli e rime a Lucrezia Borgia), O 205 sup. (De Guidubaldo Feretrio deque Elisabetha Gonzagia urbini ducibus liber), N $\mathrm{I} 26$ sup. (Oratio ad Venetos qua hortatur ut graecis litteris faveant, in greco), D 5 Is inf. (Fragmenta Historiae Venetae), P I 30 sup. (Brevia nomine Leonis PP. X), D 475 inf. (Epistulae), D 29 inf. (la Fiammetta del Boccaccio, in un manoscritto del sec. XV, con note autografe del Bembo)».

34. Travi, Pietro Bembo e il suo Epistolario cit., p. 279.

35. Pietro Aretino, Lettere, tomo I, libro I, a cura di P. Procaccioli, Roma, Salerno, i997, n. 59, pp. II4-16.

36. Pietro Aretino, Lettere, tomo I, libro I, n. 320, pp. 44I-43. La datazione della lettera nello stampato contenuto in $\mathrm{M}$ corrisponde a quella della princeps (e ristampe) delle lettere di Aretino (De le letere di M. Pietro Aretino. Libro primo, In Venetia, Appresso Francesco Marcolini, I 538 , c. Io $5 \mathrm{r}-\mathrm{v}$ ); venne poi modificata in 22 dicembre 1537 nella seconda edizione del primo libro (Del libro primo de le Lettere di M Pietro Aretino. Editione seconda con giunta de lettere XXXXIIII scrittegli da i primi spiriti del mondo, Venezia, Marcolini, I 542, pp. 474-76), seguita dal Procaccioli.

37. Cart., sec. XVIII, 200xi 50, cc. [2], pp. I-769 (numeri solo sul recto delle carte), [I], c. [I'] così numerate anticamente. Una sola mano con inserti di altra mano a colmare carte lasciate bianche. A p. [770] l'imprimatur: «Bergomi $24 \mathrm{Iu}(\mathrm{n})$ ii 1756 », confermato il 17 giugno 1757. $\mathrm{Si}$ tratta del manoscritto preparatorio per la stampa Poesie scelte dopo il Petrarca e gli altri primi, Bergamo, Lancellotti, i 756. Contiene rime di Antonio Tebaldeo, Benedetto Moreini, Vittoria Colonna, Pietro Bembo, Lodovico Ariosto, Bernardo Cappello, Giovanni Guidiccioni, Giulio Camillo Delminio, Francesco Maria Molza, Benedetto Varchi, Alessandro Piccolomini, Anton Francesco Grazzini, Fidentio Glottochrisio, Francesco Berni, Alfonso de’ Pazzi, Bernardo Tasso, Iacopo Marmitta, Girolamo Muzio, Francesco Coppetta, Anton Francesco Rainieri, Giovanni della Casa, Marco Tiene, Bernardino Rota, Annibal Caro, Domenico Veniero, Luigi Tansillo (attribuzione cassata), Torquato Tasso, Gabriello Chiabrera, Lorenzo Magalotti, Antonio Tommasi, Domenico Lazzarini, Olinto Olimpico [della seconda mano], Eustachio Manfredi, Giovan Bartolomeo Casaregi, Francesco Lorenzini, Girolamo Tagliazucchi, Fernando Antonio Ghedini, Domenico Petrosellini, Gian Antonio Gravetti (lettura incerta), Giampietro Zanotti, Francesco Maria Zanotti, Francesco Brunamonti, Carlo Innocenzo Frugoni, Ippolito Zanelli, Alessandro Fabri (attribuzione cassata), Gio. Antonio Volpi, Luigi Tanari (attribuzione cassata), Giampietro Oliva, Giulio Baiardi, Antonio Gatti (cassato), Flaminio Scarselli, Gasparo Gozzi, Giuliano d'Andrea Cassiani, Francesco Algarotti, Francesco Saverio Oliva, Giambattista Cotta, Ignazio Somai, Gaetano Passarini, Durante Duranti, Giuseppe Baretti, Giusto de' Conti, Angelo Poliziano, Giovanni Mozzarello, Ippolito de' Medici, Giambattista Amalteo, Bernardino Tomitano, Angelo Di Costanzo, Luigi Tansillo, Bernardino Baldi, Girolamo Tagliazucchi, Giambattista Ricchieri, Vincenzo da Filicaia. Tit. (c. [r] r): «Poesie scelte | Dopo il Petrarca, e gli altri | primi». Legatura in cartone con dorso in pergamena. Precedente collocazione: «Sigma IV รI».

38. Vecce, Paolo Giovio cit., pp. 92-93.

39. La trascrizione è conservativa; modifico $u$ semiconsonante in $v$, e, moderatamente, la punteggiatura secondo l'uso moderno.

40. chiar- e car- sono spesso rimanti ma più raro è il loro accostamento diretto; non ne ricorrono infatti esempi all'interrogazione delle concordanze elettroniche, l'Arcbivio della tradizione lirica, a cura di Amedeo Quondam, Roma, Lexis Progetti Editoriali, 1998, CD-Rom; e la Letteratura Italiana Zanichelli, ver. 4.0, CD-Rom dei testi della letteratura italiana a cura di Pasquale Stoppelli ed Eugenio Picchi, Bologna, Zanichelli, 200 I. 


\section{ANDrea Donnini}

4I. Dionisotti, p. 6r9: «[...]. Erano i giorni decisivi della contrastata nomina del Bembo a Cardinale: il Giovio a Roma si era adoperato a favore e il sonetto voleva essere un segno di gratitudine. Ma di lì a poco il Bembo risolse di mutare l'impianto del sonetto: nella nuova redazione, la sola conservata, gran parte ha una donna [.....».

42. Dionisotti, Appunti cit., pp. 273-74 (= ed. Vela pp. I 29-30).

43. C. Ranieri, Ancora sul carteggio tra Pietro Bembo e Vittoria Colonna, «Giornale Italiano di Filologia», XXXV (1983), p. 146, n. 41.

44. rr. I I-20: «E fo V.S. di questo sicura: che tutto ciò che ne averrà io riceverò da lui per lo migliore, e ne renderò piene grazie. Io non cercai mai d'esser Car.le, e se io n'ho a dir più oltra il vero, né anco disiderai. Non voglio già negarvi che la buona openione che ha N.S. di me avuta non mi sia gratissima stata, e più ancora per ciò che io non l'ho né mendicata né ricercata, che per altro. Ma non mi pento tuttavia di questo mio picciolo e basso stato, se non in quanto io a N.S. Dio non serva come doverei. Ma ciò nell'animo mio sta, non nella mia fortuna, e posso a S. Maestà servire così in questo stato, come in altro. [...]».

45. Il 24 marzo. Rr. I-5: «V.Ill.ma S. ha più da rallegrarsi, della nuova dignità e grado datomi da N.S., perciò che ella ne è stata in buona parte causa, che per alcun mio merito di che ella per sua molta cortesia ragione meco nelle sue lettere; [...]».

46. Con cautela si esprime Dionisotti, p. 6r 9; con maggiore certezza Dionisotti, Appunti cit., p. 270-7 I (= ed. Vela, p. I 28): «Nel sonetto l'identificazione della "donna gentil, che non perle e d'ostro, ma sol d'onor e di virtute ha sete" è probabile, perché, come si è visto, la corrispondenza letteraria fra la Colonna e il Bembo era cominciata nel i 530 con la mediazione del Giovio e perché non si sa di altra donna che a quella o a qualunque data il Bembo o il Guidiccioni o chicchessia potesse appaiare decentemente al Giovio, che non era certo noto come un donnaiolo.».

47. Negli anni in cui Bembo scrive il sonetto il rapporto fra lui e la Colonna era già mediato da Gualteruzzi.

48. Cito il commento dell'Ameyden secondo il ms. «802» della Bibl. Corsiniana di Roma; a c $292 v$ si legge il testo del sonetto, secondo la lezione di G; a c. $293 r$ la chiosa: «Invita e prega Paolo Giovio Vescovo di Nocera Historico de' suoi tempi che voglia nelle sue historie raccontar le virtù di Donna Vettoria, che di ciò ne risulterà utile a lui perché il nome di lei risplenderà nelle sue carte non meno che fa il sole nel mondo; poiché in essa sola a gara ha posto ogni suo pregio natura et arte; cosa che di rado suole avenire: che Donna di suprema bellezza et di gran valor e sapere sia cotalmente buona et d'honesti costumi. [...].». Sul commento si può vedere G. Stiénon - M. Szabò, Notice sur Théodore Ameyden - un belge - et sur ses commentaires inédits des «Rime» de P. Bembo, Bruxelles, Lamertin, I929 [estratto dai Mémoires della Reale Acc. del Belgio, Classe letter., seconda serie, t. XXVII] che non ho reperito, e la recensione di C. Dionisotti, «Giornale Storico della Letteratura italiana», XCV (I930), pp. I70-7I che a p. 170 così sintetizza la tradizione dell'opera: « $\mathrm{N}^{\circ} \mathrm{I}$, Ottobon. Lat. 226 ; autogr., completo; 2, Corsiniano 802: apografo fino a c. 390; indi autografo; correzioni e note marginali autografe in tutto il vol.; 3, Ottobon. Lat. I68 I, riassunto del $\mathrm{N}^{\mathrm{O}}$ I; 4 , Vat. Lat. 8825 , copia incompleta e difettosa del $\mathrm{N}^{\circ}$ I».

49. Annotazioni di Anton Federico Seghezzi alle Rime di M. Pietro Bembo, in Opere del Cardinale Pietro Bembo ora per la prima volta tutte in un corpo novellamente unite. Tomo secondo contenente le Prose, gli Asolani, e le Rime, con varie scritture ed illustrazioni di altri autori, come nell'indice si dichiara, In Venezia, Presso Francesco Hertzhauser Libraio all'Insegna della Roma antica, 1729, p. $214 \mathrm{~b}$. 
50. T. Zanato, Indagini sulle Rime di Pietro Bembo, «Studi di Filologia italiana», LX (2002), p. 178 .

51. Le Rime di M. Pietro Bembo. Tratte dall'essemplare riveduto, \& corretto di sua mano, tra le quali ve ne sono molte non più stampate. Con alcune annotationi poste a' suoi luoghi, così intorno alla lingua come a' concetti di Francesco Sansovino, In Venetia, Appresso F. Sansovino, MDLXI.

52. Dionisotti, Appunti cit., p. 272 (= ed. Vela, p. I 29).

53. Testimonianza della larga contezza della devozione di Giovio per la Colonna viene ad es. anche dal XXIX dei Paradossi di Ortensio Lando (ed. A. Corsaro, Roma, Edizioni di Storia e Letteratura, 2000, in Letteratura Italiana Zanichelli cit.): «[...] e a' nostri tempi qual arguto e ingegnoso poeta por si potrebbe mai al paragone della marchesana di Pescara, dell'illustre e cortese signora la signora Veronica da Gambera, o della gentil Emilia Angosciola? Non mi stenderò diffusamente in ragionare di tutte le donne che a' nostri tempi chiare sono per vera nobiltà, e riguardevoli per molta virtù, avendone di ciò copiosamente scritto monsignor Giovio vescovo in Nocciera e gran scrittor delle storie moderne.».

54. La princeps è del Is50-I 592 (Pauli Iovii Novocomensis Episcopi Nucerini Historiarum sui temporis tomus primus [-secundus], Florentiae, in officina Laurentii Torrentini ducalis typographi, I 550-I 552 ) ma l'opera era in composizione già durante il primo soggiorno romano di Giovio (dal I 5 I 4 ).

55. Vittoria Colonna, Rime, Ei

56. Paolo Giovio, Le vite del gran Capitano e del Marchese di Pescara volgarizzate da Ludovico Domenichi, a cura di C. Panigada, Bari, Laterza, I93 I, p. 495.

57. Vecce, Paolo Giovio cit., p. 86.

58. La prova della lettura dei Dialogi da parte di Bembo - come dimostra Vecce, Paolo Giovio cit., pp. $8 \mathrm{I}-83$ - risiede nella presenza sui margini del testo del Faunus bembiano trascritto da Giovio al termine del secondo dialogo (c. $2 \mathrm{I} v$ ) di varianti autografe del Bembo che risultano appartenere ad uno stadio redazionale intermedio fra il testo base del carme nel ms. Antoniano (anteriore al marzo i 5 I I) e le correzioni ivi apportate da Bembo prima del i 530. Il ms. dei primi due dialoghi si trova a Como presso la Società Storica Comense, «Fondo Aliati, cassetta 28, I-II); quello del terzo dialogo si trova sempre a Como, ma presso la Biblioteca Civica, «I. 6. I 6». I codici sono descritti da C. Vecce, Paolo Giovio cit., pp. 68-70. L'edizione critica dei Dialogi, a cura di E. Travi, si trova in Pauli Iovii Dialogi et descriptiones, curantibus E. Travi, M.G. Penco, Roma, Istituto poligrafico e Zecca dello Stato-Libreria, I984, pp. 147-321.

59. Vecce, Paolo Giovio cit., pp. 68-71.

6o. Vecce, Paolo Giovio cit., p. 75; sul contenuto dell'elogio pp. 80-8I

6r. Non è nella scelta di rime bembiane scritte dopo il I 530 raccolta da Gorni in Poeti del Cinquecento cit., pp. 213-25; né in Antologia della poesia italiana, dir. C. Segre e C. Ossola. Cinquecento, Torino, Einaudi, 2001 ${ }^{2}$ (1997).

62. D. Chiodo, Varchi rimatore: modi e forme della poesia di corrispondenza, relazione tenuta al convegno Benedetto Varchi 1503-I565, Firenze, I6-17 dicembre 2004, inedita. Ringrazio l'autore per avermela comunicata. 


\section{ANDrea Donnini}

63. Cito da Francesco Petrarca, Trionfi, Rime estravaganti, Codice degli abbozzi, a cura di V. Pacca e L. Paolino, Introduzione di M. Santagata, Milano, Mondadori, 1996.

64. Cito dall'edizione a cura di G. Dilemmi, Firenze, Presso l'Accademia della Crusca, I99I. Il luogo non varia dalla princeps ( I 505) alla seconda edizione (I 530 ) del dialogo.

65. Cito da Francesco Petrarca, Can₹oniere, edizione commentata a cura di M. Santagata, Milano, Mondadori, 1996.

66. Travi 433, rr. I3-1 8: «Mi piace molto che 'l Jovio sia costì. El qual vi dice el vero, come io stimo, a dirvi ch'egli è molto mio, perciò che io son molto suo amicissimo. Vi priego a salutarlo a mio nome, e a raccomandarmegli assai, e pregarlo a voler venir a starsi otto giorni qui con meco, a veder questo studio e li dotti che vi sono, per poter scegliere alcun nome da porre nella sua Istoria.».

67. Non compare infatti nella princeps delle Rime (Venezia, Da Sabbio i 530 ), ma nella seconda edizione (ibid., I 535 , c. 43r).

\section{Dionisotti, p. 679 .}

69. Il sonetto all'Aretino è tramandato in redazione diversa dal ms. I 387 della Biblioteca Oliveriana di Pesaro (post I 539, ante I 548), c. $56 \mathrm{r}$ (sul quale mi permetto di rinviare ad un mio contributo di prossima uscita in «Studia Oliveriana») e dalle edizioni Opera nova nella quale si contiene uno Capitulo del signor Marchese del Vasto. Stanze del signor Alvise Gonzaga. Sonetti di Monsignor Bembo, et del divino Pietro Aretino: nuovamente posti in luce, In Verona, Per Antonio Putelletto Portese. Ad instantia de G. Antonio Dento detto el Cremaschino, I 542, c. A3v, e Rime diverse di molti eccellentiss. auttori nuovamente raccolte. Libro primo, con nuova additione ristampato, In Venetia, appresso Gabriel Giolito d'i Ferrarii, i 546, p. I6; ai vv. I 2-I 4 i testimoni leggono concordi: «Ch'omai risplenderà tra le due prime / Lauretta e Bice, novo e dolce mostro / Del ciel, come Sol chiara e più sublime». Purtroppo non ricorrono informazioni sull'antigrafo utilizzato da Gualteruzzi e quindi non è possibile stabilire con piena certezza il rapporto della redazione a stampa in D con l'altra.

70. Le Rime di Giovanni Guidiccioni. Edizione critica e commentata a cura di Emilio Torchio. Tesi di dottorato in Italianistica, Università degli Studi di Padova, XVI Ciclo, son. 22 [XIX], pp. I 50-5 I. Come rileva il curatore - che ringrazio per avermi dato copia del suo lavoro - il sonetto fa sistema con i tre che lo precedono, tutti per la Colonna: da questi si evince la sua datazione, posteriore, ma non di molto, alla morte dell'Avalos (3 dicembre I 525 ).

71. Così Bembo a Cosimo Gheri in lettera da Villa Bozza, 29 ottobre i 535 con la quale invia il sonetto (Travi ${ }_{17}$ 24, rr. 23-24): «Questo Sonetto m'è nato e cresciuto, come sta, nelle vigilie di questa notte. Né io l'ho più in iscrittura posto che ora [...]». 